\title{
PROGRAM
}

\section{Third Annual Huntington Disease Clinical Research Symposium}

\author{
Organized by the Huntington Study Group
}

To be held on Saturday, 21 November 2009 in the Constellation Ballroom at the Hyatt Regency, Baltimore, Maryland, USA.

This activity has been planned and implemented in accordance with the Essential Areas and Policies of the Accreditation Council for Continuing Medical Education (ACCME) to provide continuing medical education for physicians. The University of Rochester School of Medicine and Dentistry designates this educational activity for a maximum of 3.0 credits in the AMA PRA Category 1 Credit $^{T M}$ system. Physicians should claim credit only commensurate with the extent of their participation in the activity.

The Symposium consists of three keynote addresses and four platform presentations by the following individuals, with allotted time for questions and answers after each presentation.

8:00-9:00 AM

Poster viewing.

9:00-9:10 AM

INTRODUCTION-Introduction and acknowledgments.

Jang-Ho Cha, MD, PhD. Chair, HSG Symposia Committee, and Richard Dubinsky, MD. Co-Chair, HSG Symposia Committee.

9:10-9:50 AM

Keynote AdDRESS-Huntington's Advocacy: Why I Care. Judy Roberson, RN. President, HDSA Northern California Chapter, Sacramento, CA, USA.

After nearly twenty years as an HD advocate, this widow at age 48 and mother of four tells her family's story. Although she had hoped that her efforts would save her husband, now her focus is on saving her children who are at-risk. The many years of leadership with the HDSA Northern California Chapter has served as a positive distraction, and she recommends to newcomers to find a life philosophy that will help them find meaning in this tough journey with HD.

In 1995, a strong effort was made to open an HD Clinic at UC Davis followed by being granted HSG status later that year. The clinic grew and now this HDSA Center of Excellence cares for over 200 patients with 7 studies/trials in progress.

Judy will talk about caregiving issues and the many perils to be cautioned about such as unrecognized depression and other health issues, loss of jobs, and financial worries. And the flip side of this caregiving struggle is the courage, strength, and wisdom found along the way.

Despite advances in research, the pace of enrollment in clinical trials has been disappointing. Judy will offer encouragement to families to increase participation in clinical trials, be- cause without human trials, we'll never get a treatment for HD. Along with encouragement, Judy will pass on some practical tips that she learned that helped along the caregiving journey.

Through her volunteering, she has met and worked with many outstanding, dedicated doctors, nurses, and researchers and has made many friends - to all of whom, she's grateful.

9:50-10:05 AM

Platform Presentation-Early Defect of Transforming Growth Factor $\beta 1$ Formation in Huntington's Disease. G. Battaglia, ${ }^{1}$ M. Cannella, ${ }^{1}$ B. Riozzi, ${ }^{1}$ S. Orobello, ${ }^{1}$ M.L. Maat-Schieman, ${ }^{2}$ E. Aronica, ${ }^{3}$ C. Letizia Busceti, ${ }^{1}$ A. Ciarmiello, ${ }^{4}$ S. Alberti, ${ }^{1}$ J. Sassone, ${ }^{5}$ S. Sipione,${ }^{6}$ V. Bruno, ${ }^{17}$ L. Frati, ${ }^{7}$ F. Nicoletti, ${ }^{17}$ and F. Squitieri. ${ }^{1}{ }^{1}$ IRCCS Neuromed, Pozzilli (IS), Italy; ${ }^{2}$ Leiden University Medical Center, Leiden, The Netherlands; ${ }^{3}$ University of Amsterdam, Amsterdam, The Netherlands; ${ }^{4}$ Sant'Andrea Hospital, La Spezia, Italy; ${ }^{5}$ Dino Ferrari Center, IRCCS Istituto Auxologico Italiano, Milan, Italy; ${ }^{6}$ University of Alberta, Edmonton, Canada; and ${ }^{7}$ University "Sapienza," Rome, Italy.

Background: Huntington's disease (HD) is a neurodegenerative disease due to an abnormal accumulation of mutated huntingtin with toxic effects in cortical and subcortical brain areas. Neuropathological studies have demonstrated an increased reactivity of astrocytes and oligodendroglial cells in striatum and cortex. Activation of astrocytes and microglia, which reflects a process of neuroinflammation, is already observed during the presymptomatic stage of HD. Activated astrocytes and microglia critically regulate processes of neuronal death and survival by secreting glutamate, neurotrophic factors, and pro- and antiinflammatory cytokines.

Methods: We used two transgenic animal models of HD (R6/2 and YAC128 mice), HD mouse and human brain samples and controls, cell line cultures (astrocytes cell lines and striatal knock-in cell lines), and blood serum from HD subjects.

Results: Immunohistochemical analysis of brain cortex from human HD and biochemical analysis of human serum showed a reduction of TGFB 1 levels in presymptomatic subjects, which correlate with decreased brain glucose metabolism and loss of white matter volume as assessed by nuclear magnetic resonance image analysis.

TGFB1 levels increased with the progression of disease up to the late phase of disease, being linearly associated with worsening of motor clinical scores and progression rate. As the production of TGFB1 by glial cells is under the control of mGlu3 metabotropic glutamate receptors and activation of glial mGlu3 receptors is neuroprotective via a paracrine mechanism mediated by TGFB1, we examined the ability of mGlu2/3 receptor agonists to regulate brain TGFB1 levels in mouse 
models of HD. Biochemical analysis of TGFB1 levels showed an increase of TGFB1 levels in the striatum of wild-type mice, whereas no increase was observed in presymptomatic and symptomatic R6/2 mice.

Conclusions: These data suggest that TGFB1 production could be defective in the HD brain and this could contribute to the pathophysiology of neuronal death in HD.

10:05-10:20 AM

Platform Presentation-Impairments in Precision Grip Control in Pre-symptomatic Huntington's Disease.

A. Rao, ${ }^{1}$ A. Gordon, ${ }^{2}$ and K. Marder. ${ }^{1}{ }^{1}$ Columbia University Medical Center, New York, NY, USA; and ${ }^{2}$ Teachers College, Columbia University, New York, NY, USA.

Background: Precision grip control is important for accurate object manipulation and requires coordination between horizontal (grip) and vertical (load) fingertip forces. Manifest Huntington's disease (HD) subjects demonstrate excessive and highly variable grip force, and delayed coordination between grip and load forces. Because the onset of these impairments is unknown, we examined precision grip control in presymptomatic HD (pre-HD) subjects.

Methods: Fifteen pre-HD and 15 age- and gender-matched controls performed the precision grip task in a seated position. Subjects grasped and lifted an object instrumented with a force transducer that measured horizontal grip and vertical load forces. Outcomes were preload time, loading time, maximum grip force, average grip force during the hold phase, and variability for all measures, and were computed from pre-defined events, such as onset of grip force $(>0.1 \mathrm{~N})$, onset of load force $(>0.1 \mathrm{~N})$, and maximum grip force during the trial. We measured static grip force in a 3-second window during the hold phase. Pre-HD subjects were administered the UHDRS, and predicted years to onset was calculated using a probabilistic model (Langbehn DR, et al., Clin Genet 2004;65:267-277).

Results: Preload time and loading time was slower for pre-HD subjects (158.97 ms, $123.0 \mathrm{~ms}$ ), compared with controls (64.97 $\mathrm{ms}, 57.2 \mathrm{~ms}$ ). Variability (SD) of preload and loading time was higher in pre-HD subjects $(61,55.15)$ than in controls $(35.74$, 22.08). Variability in maximum and static grip force was higher in pre-HD subjects $(0.22,0.66)$ than in controls $(0.08,0.03)$. Pearson correlation with predicted years to disease onset in pre-HD was 0.65 for loading time and 0.69 for loading time variability.

Conclusions: Temporal delay in coordination between grip and load force, and increased motor variability are seen well before diagnosis of HD and may serve as good biomarkers of disease onset and progression.

10:20-11:30 AM

Break and poster viewing.

11:30 AM-12:10 PM

KeYnOTE ADDRESS-Working Toward Mesenchymal Stem Cell Therapy for HD.

Jan Nolta, PhD. University of California, Davis, Sacramento, CA, USA.

Mesenchymal stem cells (MSC) implanted into the brain have shown strong neurorestorative effects in animal models of Huntington's disease (HD) and other neurodegenerative disorders. In the brain tissue, MSC reduce inflammation, neutralize reactive oxygen species, and diminish apoptosis to delay the loss of neurons. For the past 20-plus years, our group has studied the biosafety of human MSC engineered to produce cytokines and other factors in vivo, and we have shown sustained expression in many tissues for at least 18 months. However, low numbers of MSC cross the blood-brain barrier in our chronic disease models. Intracranial (IC) implantation of MSC and brain-derived neurotrophic factor (BDNF)-modified MSC have been shown to be safe and effective in rodent HD models. Clinical trials of MSC infusion into the brains of humans with ALS and traumatic brain injury have been done without adverse events, but therapies using this IC delivery route for MSC have not yet been initiated in the U.S.A. through FDA-approved trials. Our ongoing studies are evaluating the biosafety of MSC and then inducible BDNF-modified MSC implantation into the brains of rodents and non-human primates to generate data for the FDA in support of an initial planned phase 1 clinical trial to treat HD. We will next validate the biosafety of our patented approach to use MSC to directly deliver small interfering RNA (siRNA) into damaged and at-risk neurons for the second planned trial. SiRNA directed against mutant huntingtin mRNA are effective at ameliorating the neurodegenerative effects seen in HD and other disorders, since the mutant protein is no longer made. But it is very difficult to get siRNA across the blood-brain barrier in human patients, which has thwarted effective clincial use. We have shown that MSC can move nimbly through tissues and deliver their contents directly into target cells. Thus, for the second planned trial, we will use MSC to continually deliver anti-mutant htt siRNA directly into damaged striatal neurons, to reduce levels of mutant htt RNA and protein. Since there are currently no cures or highly effective treatments for HD, there is a high potential benefit-to-risk ratio for these studies.

\section{2:10-12:25 PM}

Platform PRESEntation-Early vs Late Huntington's Disease: Is There a Difference in Psychiatric Symptom Severity? K. Anderson, ${ }^{1}$ A. Gruber-Baldini, ${ }^{2}$ J. Medalie, ${ }^{2}$ S. Reich, ${ }^{2}$ P. Fishman, ${ }^{2}$ B. Robottom, ${ }^{2}$ W. Weiner, ${ }^{2}$ and L. Shulman. ${ }^{2}{ }^{1}$ University of Maryland, Department of Neurology \& Department of Psychiatry, Baltimore, MD, USA and ${ }^{2}$ University of Maryland, Department of Neurology, Baltimore, MD, USA.

Background: Prior to the availability of genetic testing, Huntington's disease (HD) was generally considered to become symptomatic between ages 20 and 40 years. Genetic testing has expanded the age range of HD onset and permitted the diagnosis of individuals later in life, often without an apparent family history of HD. It is not known whether the burden of psychiatric symptoms differs in patients who develop late-onset HD.

Hypothesis: Older individuals with HD have less severe psychiatric symptoms than those diagnosed earlier in life.

Methods: HD patients age 60 or over $(n=18)$ were compared with HD patients under age $60(n=27)$ using unpaired $t$-tests on self-reported psychiatric symptoms (Brief Symptom Inventory, BSI-18), and quality of life (SF-12). The Mini Mental State Examination (MMSE) was administered and global severity of HD was rated by clinicians on a 5-point Likert scale. Results: Older HD patients reported less BSI-18 depressive symptoms than younger patients $(45.7 \pm 6.4$ vs $54.3 \pm 9.8, p<$ $0.01)$. Older patients also reported less severe BSI-18 somatic symptoms, although not significant $(44.3 \pm 6.5$ vs $48.9 \pm 7.9$, $p=0.13)$, and less suicidal ideation $(p=0.08)$. The two groups did not differ on severity of anxiety symptoms. Although not significant, older patients reported lower physical health quality of life $(49.0 \pm 13.3$ vs $42.9 \pm 13.0, p=0.33)$ but better mental health quality of life $(36.8 \pm 13.8$ vs $41.7 \pm 12.5$, $p=0.23)$. There was no difference on MMSE or disease severity between groups. 
12:25-12:40 PM

Platform PResentation-Late-breaking research-Multivariate Clinical Predictors of Huntington Disease (HD): Prospective Results from the PREDICT-HD Study.

D. Langbehn, J. Paulsen, and the PREDICT-HD Investigators of the Huntington Study Group. University of Iowa, Iowa City, Iowa, USA.

Background: An original primary aim of PREDICT-HD was identification and quantification of clinical marker sets predictive of HD diagnosis. Such models would add substantial power and efficiency to eventual clinical trials to prevent or delay HD onset. Since the study's inception, skepticism has grown regarding the utility of "diagnosis" as an HD concept. For this analysis, we assume that illness transition can at least be crudely dichotomized by an abstract point of "diagnosis." Nonetheless, we see substantial evidence of inter-rater variability in defining this point and make appropriate statistical adjustments.

Methods: As of April 2009, 2179 years of pre-diagnosis follow-up were available on 718 participants, 126 of whom had been diagnosed by UHDRS confidence level 4 . We constructed multivariate models predicting "diagnosis" from the study's non-brain-imaging baseline measures. Various criteria consistently selected from among candidate variables each individually predictive of diagnosis were used. Models were tested for additional prediction beyond that achieved by CAG length and age. Cox proportional hazard modeling was used, with stratification by rater in order to minimize systematic differences in diagnostic thresholds and motor scoring.

Results: The combined baseline measures of maximum tapping speed, paced tapping precision, Stroop inference, and smell identification accuracy were highly predictive. Estimated hazard ratio increased 5.39 across the interquartile range of estimated risk $(p<0.0001)$. Adding a previously defined CAGbased predictor, smell identification lost significance. The other three variables still added substantial additional prediction. When rater motor score was added, speeded and paced tapping remained highly informative.

Conclusion: The relative risk of transition to HD "diagnosis" is predictable using measures that have played little traditional role defining diagnosis. This demonstrates important practical utility for these predictors. Absent a highly reliable, continuous measure of early HD, the success of these results also affirms the potential value of reliable discrete endpoints for early-HD trials.

\section{2:40-1:20 PM}

KEYNOTE ADDRESS-FDA Regulation of Stem Cell Therapy. Celia Witten, PhD, MD. Center for Biologics Evaluation and Research, Food and Drug Administration, Rockville, MD, USA.

Clinical trials to evaluate the safety and effectiveness of cell therapy products are regulated by the U.S. Food and Drug Administration (FDA). One of the most critical junctures in the development of a new therapeutic agent is the transition from the bench to the first clinical trials. To determine whether there is information to support initiation of a clinical trial, and whether the investigation is adequately designed to assure the safety of subjects, the FDA reviews information on the product, preclinical safety studies, and safety features incorporated into the clinical trial. Product information can include, for example, information on source controls and manufacturing process controls, as well as analytical assessment of product characteristics and safety testing for adventitious agents or other contaminants. Preclinical safety evaluation is important to establish a scientific rationale for the clinical investigation of the product and to demonstrate an acceptable safety profile of the cell therapy and its delivery system. Early stage trials are reviewed for safety. Study design aspects that enhance safety include, for example, defining an appropriate patient population, and incorporation of safety monitoring and stopping rules into the trial. Early stage trials can provide early information about safety and tolerability, as well as information on dose selection and dose delivery, proof of concept, patient selection, and information to assist in selection of clinical trial design parameters for pivotal studies. Dialogue with the FDA is encouraged during early stages of product development in order to provide investigators and sponsors with an understanding of what is needed to initiate clinical development, and at later stages to discuss what type of data would be needed to support a marketing application. 


\section{POSTER SESSION}

Posters will be staffed from 8:00-9:00 AM and 10:20-11:30 AM in the Constellation Ballroom.

\section{POSTER 1}

Caregiver Quality of Life in Huntington's Disease. M. Cox, ${ }^{1}$ A. Feigin, ${ }^{2}$ and B. Napolitano. ${ }^{3}{ }^{1}$ North Shore-Long Island Jewish Health System, New Hyde Park, NY, USA; ${ }^{2}$ The Feinstein Institute for Medical Research, North Shore-LIJ Health System, Manhasset, NY, USA; and ${ }^{3}$ Biostatistics Unit, Feinstein Institute for Medical Research, Manhasset, NY, USA.

Objective: To determine the factors that most influence the quality of life (QOL) of caregivers of persons with Huntington's disease (HD).

Background: Long-term care of individuals with HD rests mainly with family or partner caregivers, and HD care may pose unique burdens not seen in other disorders. For example, HD caregivers may need to provide various levels of care to multiple individuals over the course of their life. Identification of the factors that affect HD caregiver QOL may provide insights into easing caregiver burden.

Methods: $31 \mathrm{HD}$ caregivers $(21$ female, age $=56 \pm 11 \mathrm{yr}$, range 25-76) completed the 33-item HDQoL-C questionnaire (an unpublished QOL questionnaire for HD caregivers), provided demographic information, and responded to a single item question rating overall QOL; in addition, qualitative information was collected through two free-text questions about what factors would most improve QOL.

Results: The mean score on the questionnaire was 55\%, indicating suboptimal quality of life. The single item self-reported QOL question correlated with the total score on the HDQoL-C $(r=0.67, p<0.05)$. The number of hours spent care giving and working correlated inversely with QOL $(r=-0.43, p<$ 0.05 and $-0.43, p<0.05$, respectively). The number of times a caregiver socialized per month was positively correlated with the HDQoL-C total score $(r=0.52, p<0.01)$. Subjects most often reported in the comments of the two free-text questions that access to affordable care programs for the HD-affected person, more time for themselves, and social support would improve their QOL.

Conclusions: Providing respite from care giving specifically involving social activities may improve HD caregiver QOL. Further studies involving specific interventions to improve HD caregiver QOL are warranted.

\section{POSTER 2}

Genetic Discrimination of Individuals at Risk of Huntington's Disease: Further Analysis of the RESPOND-HD Data From Australia (Site 144).

A. Goh, ${ }^{1,2}$ O. Yastrubetskaya, ${ }^{1,2}$ and E. Chiu. ${ }^{1,2}{ }^{1}$ Academic Unit for The Psychiatry of Old Age, The University of Melbourne, Victoria, Australia; and ${ }^{2}$ St Vincent's Aged Psychiatry Service, Melbourne, Victoria, Australia.

We secured a grant from the Legal Services Board of Victoria to fund a follow-on project from RESPOND-HD to analyze and explore the Australian experience of genetic discrimination (GD), using HD as a case model. The objectives are 1) improving knowledge of laws related to GD, 2) improved legal services, and 3) development of easily accessible resources (brochure, website) informing consumers of their rights.

Results: Of 60 participants (65\% female; 35\% male; $60 \%$ HD gene positive; $40 \%$ gene negative), aged between 20 and 69 years,
$32 \%$ reported experiencing GD, with mean number of incidences 3.17 ( \pm 3.35 ). Participants experienced discrimination in employment, social, and insurance domains. For example, $17 \%$ of respondents had been refused insurance coverage, $20 \%$ offered coverage only at a higher premium, $55 \%$ told because of family history they could get only limited coverage, and $36 \%$ told that due to test results they could get only limited coverage.

Responses to Discrimination:

1. Action-getting involved in activities

2. Inaction- do nothing, react with positivity, feel surprised by discrimination

3. Involvement-information provision, research involvement

4. Advice-regarding event or potential for event

5. Advocacy - to reduce stereotyping, providing education

Awareness of Legislation: Victoria's anti-discrimination laws are contained in the Equal Opportunity Act 1995, Disability Discrimination Act 1992, and Human Rights and Equal Opportunity Commission Act 1986. Regarding GD, $17 \%$ of participants knew where to make a complaint; $27 \%$ knew of laws preventing employers from unfairly using genetic information; $15 \%$ knew of laws preventing health insurance companies from accessing or requiring genetic information about an insured person; and $10 \%$ knew of laws preventing these companies from using genetic information to deny insurance. We aim to increase these percentages. We are funded to hold focus groups to discuss results and clarify issues with several cohorts (genenegative participants, gene-positive participants, family members, case workers, health professionals, and researchers), and also for a public launch of the above-mentioned resources.

\section{POSTER 3}

Frequency of Epileptic Seizures in a Population of Juvenile Huntington's Disease Patients.

L. Cloud, S. Helmers, R. Jones, S. Factor, and C. Testa. Emory University School of Medicine, Atlanta, GA, USA.

Background: Epileptic seizures are a common feature of juvenile Huntington's disease (JHD), yet very little is known about the frequency of seizures, seizure type, electroencephalographic (EEG) characteristics, and response to anti-epileptic drugs (AEDs) in this subset of patients, much less about what correlates with increased seizure risk.

Methods and Results: Via retrospective chart review, we evaluated the frequency of seizures in our JHD population. Since inception, our clinic has seen 13 patients with genetically confirmed JHD. Incomplete medical records necessitated exclusion of three patients from our review. Of the 10 remaining patients, $5(50 \%)$ were being treated for paroxysmal events presumed to be seizures. Semiology was variable and included staring spells, hypertonicity with vocalizations, generalized convulsions, and myoclonic events. EEG data were available for 4 patients. One had a normal EEG. Two had diffuse background slowing with no obvious epileptiform abnormalities. One had a background of $2.5-4 \mathrm{~Hz}$ delta slowing that was monomorphic at times; she also had nearly continuous, bilateral parietaloccipital 2.5-3.0 Hz spike and slow wave discharges and occasional spike wave discharges seen in a more generalized distribution.

Discussion: Although the focality of the spike and slow wave discharges is suggestive of localization-related epilepsy, the presence of more generalized spike wave discharges suggests symptomatic generalized epilepsy. AEDs used in these patients 
included valproic acid, lamotrigine, levetiracetam, and alprazolam. All were managed effectively with monotherapy, except the child with epileptiform abnormalities on her EEG; she experienced an increase in her seizure frequency on levetiracetam monotherapy. Lamotrigine monotherapy was ineffective, and she is now on a combination of valproic acid and lamotrigine in attempt to reduce her seizure frequency from her current rate of 3 events per week. Expanding our understanding of the mechanism and best treatment of seizures in JHD will require analysis of data from multiple HD centers.

\section{POSTER 4}

The Effect of Video Game-Based Exercise on Dynamic Balance and Mobility in Individuals with Huntington's Disease.

D. Kegelmeyer, S. Kostyk, N. Fritz, and A. Kloos. The Ohio State University, Columbus, OH, USA.

Background: Huntington's disease (HD) is a hereditary neurodegenerative disease in which balance and gait impairments inevitably develop, placing individuals at high risk for injurious falls and lowering their quality of life.

Methods: In a study designed to examine the efficacy of an exercise program using the video game Dance Dance Revolution (DDR) to improve balance and mobility in individuals with $\mathrm{HD}$, participants will be 20 adults with HD who can ambulate 10 feet without assistance. A randomized, cross-over, control design is used. Subjects are alternately assigned to experimental $(n=10)$ and control $(n=10)$ groups. The experimental group performs DDR for 45 minutes $2 \times$ /week for 6 weeks in their homes with a researcher who provides instructions. The control group performs a hand-held video game that controls for the effects of the attention and novelty of the DDR intervention. After completing the initial intervention, the control group crosses over to the DDR intervention and the experimental group performs the hand-held video game. Compliance with the control phase and fall incidence is determined via weekly phone calls. All outcome measures including the Tinetti Mobility Test (TMT), the 4-square step test, the Activities-Specific Balance Confidence (ABC) scale, forward, backward, and obstacle course spatiotemporal gait measures using the GaitRite walkway, and subjects' reported perceptions of the video games are obtained by one of the researchers, who is blinded to group assignment.

Results and Discussion: Preliminary data analysis of subjects who have completed the study $(n=7)$ shows a trend for improvements in balance measures such as the TMT and performance on an obstacle course following the DDR intervention. The majority of subjects report that the DDR game is fun, challenging, and highly motivating, whereas the hand-held games tend to be difficult and inconvenient to play. The preliminary results indicate that DDR is well tolerated, and may improve balance in this population.

\section{POSTER 5}

Usefulness of Two Brief Cognitive Screening Measures in Huntington's Disease.

S. Lessig, J. Goldstein, and J. Corey-Bloom. University of California, San Diego, San Diego, CA, USA.

Objective: To analyze performance and rate of change on two brief cognitive measures in Huntington's disease (HD).

Background: Little is known about tracking change on brief cognitive tests in HD. The Mini-Mental Status Examination (MMSE) and the Montreal Cognitive Assessment (MoCA) are two cognitive measures of 30 points each that take about 10 minutes to administer.

Methods: HD subjects $(n=49)$ were tested on both the MMSE and MoCA in counterbalanced order at baseline and approximately 1 year later. Subjects who declined were compared with those who did not on multiple clinical features.

Results: The overall group of HD subjects showed a mean age of $50.8(\mathrm{SD}=12.9)$ yrs, education of $13.9(\mathrm{SD}=2.5) \mathrm{yrs}$, Independence Scale score of $70.5(\mathrm{SD}=11.3)$, and $\mathrm{CAG}$ repeats of $44.7(\mathrm{SD}=3.9)$. Mean baseline scores were 24.5 $(\mathrm{SD}=3.5)$ on the MMSE and $20.0(\mathrm{SD}=5.1)$ on the MoCA. The annualized rate of change for HD subjects was 0.72 points per year on the MMSE and 1.1 points per year on the MoCA. Approximately one half of subjects did not decline over time. Of those who did, annualized rates of change were 2.3 points on the MMSE and 2.5 points on the MoCA. When subjects who declined were compared with those who did not, there were no significant differences with regard to most clinical and demographic features (including age, education, CAG repeats, UHDRS motor score, baseline MMSE or MoCA scores). Only the Independence Scale suggested a difference between those who declined $($ mean $=67.0 ; \mathrm{SD}=9.8)$ on the MMSE $v$ s those who did not $($ mean $=74.3 ; \mathrm{SD}=12.1, p=0.026)$.

Conclusions: Both the MMSE and MoCA appear to have utility as brief cognitive instruments in HD. Surprisingly, although the MoCA may be better for detecting cognitive deficits, the MMSE, which better reflects functional decline, may be more useful for tracking disease progression.

\section{POSTER 6}

Cerebrospinal Fluid (CSF) Volume Is the Main Marker of Huntington Disease Progression Severity and Age-at-Onset Prediction.

F. Squitieri, ${ }^{1}$ M. Cannella, ${ }^{1}$ M. Simonelli, ${ }^{1}$ T. Martino, ${ }^{1}$ C Colonnese, ${ }^{1}$ and A. Ciarmiello. ${ }^{2}{ }^{1}$ IRCCS Neuromed, Pozzilli (IS), Italy; and ${ }^{2}$ Sant'Andrea Hospital, La Spezia, Italy.

Background: Searching brain and peripheral biomarkers is a requisite to cure for Huntington's disease (HD). Mutated huntingtin exerts its main pathological effects on brain neurons. Neuronal dysfunction and degeneration cause progressively invalidating extrapyramidal symptoms, cognitive decline, and behavioral changes. Neuropathological studies showed progressive striatal dysfunction and degeneration since the beginning of the disease.

Methods: We analyzed the cross-sectional and longitudinal rate of brain atrophy, quantitatively measured by fully automated multiparametric magnetic resonance imaging, as fractional gray matter (GM, determining brain cortex volume), white matter (WM, measuring the volume of axonal fibers), and corresponding cerebrospinal fluid (CSF, a measure of global brain atrophy), in 94 gene-positive subjects with pre-symptomatic to advanced HD and age-matched healthy controls.

Results and Discussion: Each of the three brain compartments we studied (WM, GM, and CSF) had a diverse role, and their time courses differed in the development of HD. GM volume decreased early in life. Its decrease was associated with decreased serum brain derived neurotrophic factor and started even many years before onset symptoms, then decreased slowly in a nonlinear manner during the various symptomatic HD stages. WM volume loss also began in the pre-symptomatic stage of HD, a few years before manifest symptoms appear, rapidly decreasing near to the zone-of-onset. Finally, the CSF volume increase began many years before age at onset. Its volume measured in pre-symptomatic subjects contributed to 
improving the CAG-based model of age at onset prediction. The progressive CSF increase depended on CAG mutation size and continued linearly until the last stages of HD since the pre-symptomatic life stage, representing the best marker of progression rate and severity in $\operatorname{HD}\left(R^{2}=0.25, p<0.0001\right)$ and improving the CAG-based prediction of age at onset.

\section{POSTER 7}

Early Defect of Transforming Growth Factor $\beta 1$ Formation in Huntington's Disease.

G. Battaglia, ${ }^{1}$ M. Cannella, ${ }^{1}$ B. Riozzi, ${ }^{1}$ S. Orobello, ${ }^{1}$ M.L. Maat-Schieman, ${ }^{2}$ E. Aronica, ${ }^{3}$ C. Letizia Busceti, ${ }^{1}$ A. Ciarmiello, ${ }^{4}$ S. Alberti, ${ }^{1}$ J. Sassone,${ }^{5}$ S. Sipione, ${ }^{6}$ V. Bruno, ${ }^{17}$ L. Frati, ${ }^{7}$ F. Nicoletti, ${ }^{17}$ and F. Squitieri. ${ }^{1}{ }^{1}$ IRCCS Neuromed, Pozzilli (IS), Italy; ${ }^{2}$ Leiden University Medical Center, Leiden, The Netherlands, ${ }^{3}$ University of Amsterdam, Amsterdam, The Netherlands; ${ }^{4}$ Sant'Andrea Hospital, La Spezia, Italy; ${ }^{5}$ Dino Ferrari Center, IRCCS Istituto Auxologico Italiano, Milan, Italy; ${ }^{6}$ University of Alberta, Edmonton, Canada; and ${ }^{7}$ University "Sapienza," Rome, Italy.

For text of abstract, see platform presentation (above, 9:5010:05 AM).

\section{POSTER 8}

Reliability of the Problem Behaviours Assessment for HD (short version)-Data From the TRACK-HD Study.

J. Callaghan, ${ }^{1}$ N. Arran, ${ }^{1}$ M.F. Boisse, ${ }^{6}$ A. Coleman, ${ }^{3}$ R. Dar Santos, ${ }^{3}$ E. Dumas, ${ }^{2}$ E. 't Hart, ${ }^{2}$ D. Justo, ${ }^{5}$ J. Read, ${ }^{4}$ M. Say, ${ }^{4}$ S. Van Den Bogaard, ${ }^{2}$ A. Durr, ${ }^{5}$ B. Leavitt, ${ }^{3}$ R. Roos, ${ }^{2}$ S. Tabrizi, ${ }^{4}$ C. Bourdet, ${ }^{6}$ E. van Duijn, ${ }^{2}$ D. Craufurd, ${ }^{1}$ and the TRACK-HD Investigators. ${ }^{I}$ Department of Genetic Medicine, Central Manchester University Hospitals Foundation NHS Trust and University of Manchester, Manchester, UK, ${ }^{2}$ Department of Neurology, Leiden University Medical Centre, Leiden, The Netherlands; ${ }^{3}$ Department of Medical Genetics, University of British Columbia, Vancouver, British Columbia, Canada; ${ }^{4}$ Institute of Neurology, University College London, London, UK, ${ }^{5}$ Department of Genetics and Cytogenetics, and INSERM UMR S679, APHP Hôpital de la Salpêtrière, 75013 Paris, France; and ${ }^{6}$ Service de Neurologie, CHU Henri MondorAlbert Chenevier, AP-HP, Unité Inserm 955, Equipe 1, Institut Mondor de Recherche Biomédicale, Créteil, France.

Background: TRACK-HD is a multi-center, longitudinal study investigating a range of motor, cognitive, neuropsychiatric, and imaging measures as potential biomarkers for clinical trials in Huntington's disease (HD). Psychiatric assessments were conducted using a 10-item semi-structured interview, the short version of the Problem Behaviours Assessment for HD (PBA-s). Interviews were video recorded for quality assurance purposes, and a proportion randomly selected for re-scoring at another site. The study therefore provided an opportunity to examine the reliability of the measure between multiple pairs of raters in different cultural and linguistic contexts.

Methods: The study included 360 subjects (120 affected, 120 premanifest gene carriers, 120 controls), and 185 interviews were selected for re-scoring by an expert rater. Digital video recordings were uploaded to the TRACK-HD Web portal and selected for re-scoring by an independent investigator blind to the subjects' diagnosis. A further 73 interviews were obtained from the REGISTRY clinic in Manchester and used for training the expert raters.

Results: Complete agreement between raters was achieved for $86 \%$ of scores $(\kappa=0.733)$, and $97.6 \%$ were within one point of the expert rating, the standard for quality assurance purposes. Agreement levels for individual pairs of raters ranged from $75 \%$ to $94 \%(\kappa=0.59-0.86)$; agreement within one point ranged from $92 \%$ to $99 \%$. Complete agreement for the 20 individual ratings (10 symptoms * frequency and severity) ranged from $67 \%$ to $100 \%(\kappa=0.56-0.97)$; agreement within one point ranged from $94 \%$ to $100 \%$.

Conclusions: The overall results indicate substantial agreement between all raters, whereas the $\kappa$-statistic for individual rater pairs ranged from 'moderate' to 'almost perfect' agreement, with almost all raters achieving at least a 'substantial' level of agreement. These data suggest that the PBA-s, with appropriate training, is a reliable measure of psychiatric symptoms in the HD population.

\section{POSTER 9}

The Short Version of the Problem Behaviours Assessment for HD (PBA-s): An Item Response Analysis Using Data from the TRACK-HD Study.

K. Evans, ${ }^{1}$ A. Vaccarino, ${ }^{1}$ D. Craufurd, ${ }^{2}$ J. Callaghan, ${ }^{2}$ A. Durr, ${ }^{3}$ B. Leavitt, ${ }^{4}$ R. Roos, ${ }^{5}$ S. Tabrizi, ${ }^{6}$ and the TRACK-HD Investigators. ${ }^{1}$ Ontario Cancer Biomarker Network, Toronto, Ontario, Canada; ${ }^{2}$ Department of Genetic Medicine, Central Manchester University Hospitals Foundation NHS Trust and University of Manchester, Manchester, UK; ${ }^{3}$ Department of Genetics and Cytogenetics, and INSERM UMR S679, APHP Hôpital de la Salpêtrière, 75013 Paris, France; ${ }^{4}$ Department of Medical Genetics, University of British Columbia, Vancouver, British Columbia, Canada; ${ }^{5}$ Department of Neurology, Leiden University Medical Centre, 2300RC Leiden, The Netherlands; and ${ }^{6}$ Institute of Neurology, University College London, Queen Square, London, UK.

Background: TRACK-HD is a multi-center, longitudinal study investigating a range of motor, cognitive, neuropsychiatric, and imaging measures as potential biomarkers for clinical trials in Huntington's disease (HD). Psychiatric assessments were conducted using a 10-item semi-structured interview, the short version of the Problem Behaviours Assessment for HD (PBA-s). To better characterize the relationship between scores on individual PBA items and overall severity of behavioral symptoms, a nonparametric item response analysis (Ramsay JO, Psychometrika 1991;56:611-630) was performed on the data from year 1 of the study.

Methods: The study included 366 subjects (123 affected, 123 premanifest gene carriers, 120 controls). Option Characteristic Curves (OCCs) were generated for all PBA-s items to determine the probability of scoring a particular option in relation to total PBA score.

Results: Most items, including Depressed Mood, Anxiety, Irritability, Aggressive Behavior, and Apathy showed generally good discriminative properties, but differences were observed between the three populations (affected, pre-manifest, and control subjects) in terms of their IRT curves. Suicidality was primarily endorsed at the higher levels of overall severity and did not discriminate well at lower PBA scores. A number of symptoms were rarely endorsed in this population at any level of overall behavioral symptom severity, including Delusions, Hallucinations and Disorientation.

Conclusions: These results demonstrate that some items on the PBA-s are more sensitive than others in capturing the overall severity of behavioral symptoms in early and pre-manifest HD, and thus may be more suitable to provide a reliable and valid measure of behavioral symptoms in this population. 


\section{Poster 10}

Degree of Disability Is Predictive of Physical But Not Mental Health Quality of Life in Huntington's Disease. B. Robottom, ${ }^{1}$ K. Anderson, ${ }^{13}$ J. Medalie, ${ }^{1}$ A. Gruber-Baldini, ${ }^{2}$ P. Fishman, ${ }^{1}$ S. Reich, ${ }^{1}$ W. Weiner, ${ }^{1}$ and L. Shulman. ${ }^{1}{ }^{1}$ University of Maryland School of Medicine, Department of Neurology, Baltimore, MD, USA; ${ }^{2}$ University of Maryland School of Medicine, Department of Epidemiology and Preventive Medicine, Baltimore, MD, USA; and ${ }^{3}$ University of Maryland School of Medicine, Department of Psychiatry, Baltimore, MD, USA.

Objective: To determine whether self-reported measures of disability are predictive of health-related quality of life (QoL) in Huntington's disease (HD).

Background: The disability paradox is a term coined to describe patients who report high quality of life despite chronic disability. Patients' objective health status and presence of disability may not correlate with their perception of healthrelated quality of life. This disability paradox has not been examined in patients with HD.

Methods: HD patients $(n=25)$ completed self-reported measures of disability (the Older Americans Resource and Services ADL/IADL Scale, or OARS) and health-related QoL (SF-12 Health Status Survey, Physical and Mental Health Summary Scores). Clinician ratings of disease severity were collected using a five-point Likert scale. Pearson's correlations were used to analyze the relationship between level of disability, healthrelated QoL, and disease severity.

Results: Greater disability was associated with lesser physical health QoL (OARS Total $r=0.57, p<0.05$; OARS ADL $r=$ $0.53, p<0.05$; OARS IADL $r=0.58, p<0.05$ ) and greater disease severity $(r=0.87, p<0.001)$. The OARS ADL subscale was weakly associated with mental health QoL $(r=$ $0.44, p<0.05)$. Total OARS and the OARS IADL subscale did not correlate with mental health QoL $(r=0.41, p=0.053 ; r=$ $0.38, p=0.075)$. Greater disease severity correlated with physical health QoL $(r=0.50, p<0.05)$ but not mental health QoL $(r=0.33, p=0.17)$.

Conclusions: The degree of self-reported disability in HD correlates with self-reported physical health QoL and clinicianrated disease severity but not with self-reported mental health QoL. This example of the disability paradox reinforces the need to evaluate and treat both physical and mental symptoms of $\mathrm{HD}$, as each may contribute independently to quality of life.

\section{Poster 11}

Striatal Volume Distinguishes Converters from Non-Converters: Findings from PREDICT-HD.

E. Aylward, ${ }^{1}$ P. Nopoulos, ${ }^{2}$ C. Ross, ${ }^{3}$ R. Pierson, ${ }^{2}$ J. Mills,${ }^{2}$ D. Langbehn, ${ }^{2}$ V. Magnotta, ${ }^{2}$ H. Johnson, ${ }^{2}$ J. Paulsen, ${ }^{2}$ and the PREDICT-HD Investigators and Coordinators of the Huntington Study Group. 'Seattle Children's Research Institute, Seattle, WA, USA; ${ }^{2}$ University of Iowa, Iowa City, IA, USA; and ${ }^{3}$ Johns Hopkins University, Baltimore, MD, USA.

Objective: To present data from 32 converter participants and 80 matched non-converter participants from the PREDICT-HD study.

Methods: All participants were prodromal when they enrolled in the study and all were examined yearly for at least one visit following enrollment. Converters were defined as those individuals who, on at least two consecutive visits, received a score of 4 on the HD Diagnostic Confidence Scale of the UHDRS. Non-converters were individuals who had at least two visits and never received a score of 4 . Converters and non-converters were group-matched on age, gender, and CAG repeat length. MRI measurements were obtained for 4 converters 2 years before diagnosis, for 11 converters 1 year before diagnosis, and for 17 converters at the same visit that the diagnosis was made. Results and Discussion: After correcting for intracranial volume (ICV), converters had significantly smaller total brain volume $(p=0.001)$, total white matter volume $(p=0.004)$, and total striatum volume $(p<0.0001)$, but did not differ on size of cerebellum. A logistic regression model was performed with age and gender included first, with the model selecting the remaining variables one by one. The ratio of striatum/ICV was the only variable selected yielding significant discrimination between converters and non-converters $(p<0.0001)$, with correct classification of $78.2 \%$ of participants. None of the other MRI measures provided additional significant predictive value to the model. Results indicate that striatal volume, as assessed on MRI, but not other brain measures examined, contributes additional information beyond age and CAG repeat length in predicting onset of diagnosable HD signs. This information, assuming replication in larger samples, highlights the importance of striatal atrophy in the neurobiology of HD, and will be useful for designing clinical trials in which diagnosis of $\mathrm{HD}$ is the primary outcome measure.

\section{Poster 12}

Self-Efficacy in Health Behaviors: Is There a Difference in Huntington vs Parkinson Disease?

A. Mitchell, ${ }^{1}$ K. Anderson, ${ }^{2}$ J. Medalie, ${ }^{3}$ A. Gruber-Baldini, ${ }^{3}$ B. Robottom, ${ }^{3}$ P. Fishman, ${ }^{3}$ S. Reich, ${ }^{3}$ W. Weiner, ${ }^{3}$ and L. Shulman. ${ }^{3}{ }^{1}$ University of Maryland, School of Medicine, Department of Pediatrics, Division of Human Genetics, Baltimore, MD, USA $;{ }^{2}$ University of Maryland, Department of Neurology \& Department of Psychiatry, Baltimore, MD, USA; and ${ }^{3}$ University of Maryland, Department of Neurology, Baltimore, $M D$, USA.

Background: Self-efficacy is defined as individuals' perception of their ability to successfully perform certain tasks or behaviors, or more specifically, as the belief that one can carry out a behavior to achieve a desired goal related to one's health. Higher self-efficacy is correlated with better outcomes in pulmonary disease and fibromyalgia. The role of self-efficacy in disease management has not been examined in Huntington disease (HD).

Hypothesis: Individuals with HD have lower self-efficacy for disease self-management than those with Parkinson disease (PD), because of more severe HD clinical course.

Methods: Both HD and PD patients were administered the Lorig self-efficacy scale, Mini Mental State Exam (MMSE), Brief Symptom Inventory (BSI-18, psychiatric assessment), and a demographics questionnaire. Global disease severity was clinician-rated using a five-point Likert scale.

Results: The sample comprised $22 \mathrm{HD}$ patients and $947 \mathrm{PD}$ patients. The HD patients were younger (54.4 \pm 13.0 vs $66.4 \pm$ $11.0, p=0.01)$, scored worse on the MMSE (24.74 \pm 3.56 vs $28.06 \pm 2.93, p<0.01)$, and were less educated $(p=0.016)$. The two groups did not differ significantly in gender ratio. HD patients reported lower self-efficacy than PD patients in 2 of the 10 Lorig subscales: Ability to Obtain Disease Information (1.94 \pm .75 vs $2.29 \pm .69, p=0.04)$ and Effective Communication with Physicians $(7.35 \pm 1.73$ vs $8.07 \pm 1.32, p=0.03)$. There was not a significant difference between groups on reported psychiatric symptom severity, but there was a trend toward HD patients reporting greater depression and anxiety (53.59 \pm 14.22 vs $51.34 \pm 9.77, p=0.35 ; 56.18 \pm 14.54$ vs $52.47 \pm$ 9.74, $p=0.13)$. 
Conclusions: HD patients show lower self-efficacy in certain, but not all, health behaviors. Greater degree of cognitive decline in the HD group, reflected in MMSE scores, may have played a role in perception of self-efficacy for various domains. Because self-efficacy is a modifiable factor, enhancing HD patients' self-efficacy in those targeted areas with reported deficits may improve HD outcomes.

\section{POSTER 13}

Huntington's Disease Disrupts Motor Control Mechanisms That Rely on Internal Models.

J.C. Cortes, A. Dahmubed, K. Marder, J. Krakauer, and P. Mazzoni. Columbia University, New York, NY, USA.

Background: Previous studies demonstrated that Huntington's disease (HD) impairs the ability to perform to perform online adjustments during reaching movements. It is not clear whether the difficulty is in responding to errors in observed or predicted trajectory. Do patients have trouble observing deviations of the hand from its goal, or does the difficulty lie in predicting where the hand should be based on internal models of the effects of motor commands?

Methods: We compared corrections made to two visuomotor perturbations that were equivalent in sensory space, but differed in the nature of the resulting error (goal-related $v s$ effector-related). Subjects made planar reaching movements to guide a screen cursor to a target. Interspersed with baseline trials were perturbation trials, in which either the target jumped to a new location (target jump) or the direction of the cursor was rotated relative to the starting point (rotation). The target jump (to a new direction, \pm 30 degrees away) and the rotation ( \pm 30 degrees) were spatially matched so as to require the same change in hand movement direction in order to acquire the target. The target jump required a change in movement goal (external error), whereas rotation introduced a mismatch between actual hand position and hand position predicted by subject's visuomotor map (internal model error).

Results and Discussion: The corrections made by patients to target jumps were only slightly abnormal, with increased variability and slight undershoot. Their corrections to visuomotor rotation trials, on the other hand, were considerably abnormal. There was a systematic delay in the onset of the correction, and the amplitude of the correction was incomplete. These findings establish a selective impairment in monitoring errors in computation of predicted hand position. A function of the circuits affected by HD may be to monitor internal motor control models that are necessary to guide movements to their targets.

\section{POSTER 14}

Evaluation of Novel Radiotracers Targeting Non-Dopaminergic Striatal Biomarkers in HD: ${ }^{18}$ F-FPEB and PET Imaging for Metabotropic Glutamate Receptor Type 5 (mGluR5) Expression in Healthy Subjects and Subjects with Huntington Disease (HD).

D. Russell, D. Jennings, G. Tamagnan, J. Seibyl, A. Koren, G. Zubal, and K. Marek. The Institute for Neurodegenerative Disorders and Molecular NeuroImaging, New Haven, CT, USA.

Objective: Presented here is a first-in-human study of 3- $\left[{ }^{18} \mathrm{~F}\right]$ fluoro-5-(2-pyridinylethynyl)benzonitrile ( $\left.{ }^{18} \mathrm{~F}-\mathrm{FPEB}\right)$ and PET imaging of mGluR5 in subjects with HD and healthy controls

Background: mGluR5 is highly expressed on enkephalinergic striatal neurons and in other neocortical structures. mGluR5 expression may be reduced because of neuron loss or alteration of gene expression. Therefore, mGluR5 may be a useful biomarker for early brain changes in HD, HD progression, or for drug development in HD. ${ }^{18}$ F-FPEB binds mGluR5 with high affinity and specificity. Prior studies in non-human primates demonstrated ${ }^{18}$ F-FPEB uptake consistent with the known distribution of mGluR5 expression.

Methods: Early HD patients $(n=2)$ and healthy controls (HC, $n=2$ ) underwent imaging. Subjects received $5 \mathrm{mCi}{ }^{18} \mathrm{~F}-\mathrm{FPEB}$ i.v. and serial dynamic PET projection data were acquired for 3 to 6 hours. Regional equilibrium tissue distribution volume measurements were assessed in striatal, neocortical, thalamic, and cerebellar regions. The standardized uptake value (SUV) ratios of targets to a cerebellar reference region were compared in $\mathrm{HD}$ and $\mathrm{HC}$.

Results: Both HD subjects were female with Htt repeat expansions $\geq 35$. HD_1: age $54 \mathrm{yr}$, UHDRS subscores motor 13 and TFC 13. HD_2: age $44 \mathrm{yr}$, UHDRS motor 6 and TFC 11. The HCs were both male, ages 57 and 43 yr. The HCs revealed intense uptake in striatal and cortical gray matter areas, moderate uptake in thalamus, and low, but not absent, uptake in cerebellum. For HCs, mean SUV ratios to cerebellum were, for anterior cingulate, 6.79; for temporal lobe, 6.15; for caudate, 5.44; and for putamen, 4.89. The HD subjects revealed a mean reduction in ${ }^{18} \mathrm{~F}$-FPEB uptake in striatal regions of $25-30 \%$ and temporal lobe and limbic cortical areas of $20-35 \%$.

Conclusions: ${ }^{18} \mathrm{~F}-\mathrm{FPEB}$ is a promising PET radiotracer for evaluating mGluR5 with excellent characteristics for human imaging. This first-in-human study indicates that ${ }^{18} \mathrm{~F}-\mathrm{FPEB}$ PET may detect early reductions in striatal and neocortical mGluR5 in relatively mildly symptomatic HD subjects. Future studies are ongoing to evaluate these objectives and other nondopaminergic striatal markers further.

\section{POSTER 15}

A Successful Short- and Long-Term Recruiting Strategy for Huntington Clinical Trials: A Community Effort.

L. Goodman, ${ }^{1}$ R. Carnes, ${ }^{2}$ J. Giuliano, ${ }^{3}$ and L. Vetter. ${ }^{4}{ }^{1} H D$ Drug Works, Seattle, WA, USA; ${ }^{2}$ Olympia Support Group, Olympia, WA, USA; ${ }^{3}$ CHDI Foundation, Princeton, NJ, USA; and ${ }^{4}$ Huntington's Disease Society of America, New York, NY, USA.

Objective: To determine short- and long-term effects on recruitment rates following community-delivered education sessions within a cohort of Huntington disease (HD) support group members in the Pacific Northwest.

Background: There has been no systematic measure or comparison of methods utilized in the recruitment of participants to clinical trials.

Methods: One year prior to this study, a clinical research education session was given by a community member to HD support groups in the Pacific Northwest. These sessions were purposely low tech and conversational, and questions were encouraged. Participants were made aware of the availability of TRACK-HD during those sessions. One year later, they were made aware of the upcoming local availability of the HART clinical trial. The number of recruits for TRACK-HD and HART was recorded.

Results: Just following the education sessions, 6 support group members were recruited to TRACK-HD, which was not offered locally but required the extra effort of several hours of travel and an overnight stay. This represented a $75 \%$ rate for recruitment of those meeting study criteria. One year later, without any refresher or follow-up education session, 12 support group members who met criteria for HART were ready to sign up on 
the first day of official recruitment. This would represent a $75 \%$ recruitment rate.

Conclusions: One to two hours of community-delivered clinical research education in support groups is a successful recruitment strategy on both the short and the long term. Although numbers in this project were small, the results suggest that a grass-roots, low-tech, and conversational delivery can improve rate of recruitment.

\section{POSTER 16}

Genetic Approach to Huntington's Disease Therapeutics. M. MacDonald, J. Min Lee, I. Sik Seong, E. Fossale, M.A. Anderson, V. Wheeler, and J. Gusella. Center for Human Genetic Research, Massachusetts General Hospital, Boston, MA, USA.

Background: Unlike other neurodegenerative disorders, Huntington's disease, with its motor, behavioral, and cognitive deficits and early loss of neurons in the striatum, has a single cause: expanded versions of a polymorphic CAG repeat in the $H T T$ gene (previously $H D$ ) of $\geq 35$ units. This uniform starting point permits the development of therapeutics based solely upon the features of the HTT CAG repeat.

Methods, Results, and Discussion: We are applying global methods, such as genome-wide gene expression levels, to discover dominant and CAG length-dependent genes and pathways, using cells and tissues bearing the full-range of HTT CAG allele sizes. These phenotypes can potentially point to small molecule or gene modifiers and can be converted into cell-, pathway- or gene-based assays suitable for high-throughput screens to find modifiers of HTT CAG size-dependent effects in models and humans. This genetic approach to therapeutic target discovery has demonstrated that the HTT CAG repeat is a functional polymorphism that, over the non-HD and the HD ranges, affects energy, RNA metabolism, and the cytoskeleton. These HTT CAG size-dependent signatures provide an unbiased read-out for assays with which to find compounds or genes that may slow or halt the earliest phase of the HD disease process.

\section{POSTER 17}

Metabolic Profiling in Non-Human Primate Model of Huntington's Disease Reveals Early Disruptions in Tryptophan Metabolism.

J. Johnson, T. Yu, D. Jones, and A. Chan. Emory University, Atlanta, GA, USA.

Background: Metabolomics is the systematic study of small molecule metabolites that are the end product of all forces, both endogenous and exogenous, that act upon a specific organism. Interactions among diet, environment, and genes, and their relative contribution to human health and disease, can be elucidated using metabolomics. Although rodent models of HD have been developed, these models do not satisfactorily parallel the brain changes and behavioral features observed in HD patients.

Methods: Hallmark features of HD, including nuclear inclusions and neuropil aggregates, were observed in the brains of transgenic monkeys expressing the huntingtin gene. Additionally, the transgenic monkeys showed important clinical features of HD, including dystonia and chorea. Taking advantage of new technology, we developed a method that uses a drop of blood processed with a simple extraction method to remove the proteins and injects directly onto a liquid chromatographyFourier transform mass spectrometry (LC-FTMS) system. In 10 minutes, we are able to reliably detect 2500 metabolites. The method was applied to plasma from 2 HD monkeys (birth to 8 months) and wild-type controls.

Results and Discussion: Of the metabolites detected, 19 varied significantly between HD and wild type, 2 of which were important in tryptophan metabolism. These results confirm clinical research showing a disruption in tryptophan metabolism in HD patients. The 32 metabolites that changed most with time were compared with these 19 metabolites, and 2 unknown metabolites were found on both lists. Further investigation into the identity of these metabolites revealed one with a core indole group, suggesting an uncharacterized metabolite of tryptophan. Comparison of transgenic and wild-type monkeys showed putative biomarkers of early disease with relatively few animals. A pilot study is currently underway to test these results in humans and to suggest future therapeutic targets for drug development. 


\section{LATE-BREAKING RESEARCH}

\section{POSTER 18}

Lack of Awareness of Motor and Cognitive Phenoconversion in Huntington's Disease.

K. Richardson, ${ }^{1}$ E. McCusker,${ }^{1}$ C. Loy, ${ }^{1}$ J. Griffith, ${ }^{1}$ J. Mills, ${ }^{2}$ J. S. Paulsen, ${ }^{2}$ and the PREDICT-HD Investigators of the Huntington Study Group. ${ }^{I}$ Westmead Hospital, Sydney, NSW, Australia; and ${ }^{2}$ University of Iowa, Iowa City IA, USA.

Background: Self-awareness of Huntington's disease (HD) onset has rarely been studied in a premanifest cohort, but poor recognition and reporting of symptoms could lead to a delay in diagnosis and, with that, appropriate management/care and notification of family regarding their HD risk. Moreover, poor awareness of first onset of HD could result in collecting inaccurate information about the disease; treatment, if available, could be delayed; and reports of drug effectiveness, inaccurate. Also, some patients, once they have undergone testing for the HD mutation, become overly "aware" of possible symptoms. We investigated this in a cross-sectional sample of 615 participants attending their third visit in the Predict-HD study.

Results: Of these, 79 (13\%) were recently diagnosed with manifest HD. However, more than a third of those diagnosed did not acknowledge any disease symptoms, despite showing similar signs of decline in terms of UHDRS Motor and TFC scores, psychomotor performance (Tapper), and companion ratings of frontal/executive dysfunction. Participants who reported not noticing any symptoms of HD also reported significantly less depression and fewer behavioral difficulties (indicative of frontal/executive dysfunction), consistent with poor insight. For participants who were classified as premanifest, those reporting symptoms of HD tended to have more cognitive, psychiatric, and other symptoms (compared to motor symptoms, which were most common in the recently diagnosed group). In the premanifest group, participants reporting symptoms had significantly higher ratings of depression and frontal/ executive dysfunction (compared to those who were 'symptomfee'), rating themselves similarly to the group with HD diagnosis, despite fewer signs of disease (i.e., better Motor, TFC, and Tapper scores) and longer estimated time to disease onset.

Conclusions: These results suggest that poor awareness of HD is a substantial problem in the premanifest or early stages of $\mathrm{HD}$, but further research is needed to examine the predictors and implications of under/over reporting of HD symptomatology.

\section{POSTER 19}

Validation of the Modified Motor Score (mMS): A Subscale of the Unified Huntington's Disease Rating Scale (UHDRS) Motor Score.

S. Waters, ${ }^{1}$ J. Tedroff, ${ }^{2}$ and K. Kieburtz. ${ }^{3}{ }^{1}$ NeuroSearch Sweden AB, Göteborg, Sweden; ${ }^{2}$ NeuroSearch Sweden AB, Stockholm, Sweden; and ${ }^{3}$ University of Rochester, Rochester, NY, USA.

Background: The UHDRS assesses various aspects of clinical function and capacity in Huntington's disease (HD). Items in the UHDRS motor section are heterogeneous, relate to various aspects of HD motor symptoms, and can be categorized as eye, involuntary, or voluntary movements. Items relating to voluntary movements appear to correlate strongly with the extent of disability, whereas the correlation between chorea and disability is less pronounced. These findings are supported by a pre- liminary analysis of baseline data from a phase 2 study of pridopidine (ACR16) in patients with HD (ACR16C007).

Methods: Motor scales from the UHDRS motor section were analyzed according to their metric properties and correlation with measures of functional disability, assessed by total functional capacity (TFC) and functional assessment (FA) scales. The following motor scales were analyzed, using data from the CARE-HD study dataset: UHDRS motor section items (TMS); TMS excluding eye movements; modified motor scale (mMS: TMS excluding eye movements, chorea, and dystonia), which assessed voluntary movements; chorea; and eye movements. Results: The mMS subscale correlated strongly with TMS ( $\rho$ $=0.89 ; p<0.0001)$. For functional disability measures, mMS correlated slightly more strongly with FA than the TMS did ( -0.53 vs. -0.52 , respectively), whereas chorea and eye movements correlated less strongly. The mMS showed improved internal consistency compared with the TMS, and intra-class correlation for the mMS was similar to that for the TMS $(\sim 0.90)$, indicating good test-retest reliability. Intra-class correlations for chorea and eye movements were lower $(\sim 0.80)$. Conclusions: The mMS subscale appears to be a valid modification of the UHDRS motor score and has functional relevance, as demonstrated by strong correlations with functional assessments. This subscale of the UHDRS motor sections may offer a simple method of assessing response to treatment in patients with HD.

\section{POSTER 20}

Pridopidine (ACR16) in Huntington's Disease: An Update on the MermaiHD and HART Studies.

J. Tedroff, ${ }^{1}$ P. Lindskov Krogh, ${ }^{2}$ A. Buusman, ${ }^{2}$ and $\AA$. Rembratt. ${ }^{2}{ }^{1}$ NeuroSearch Sweden AB, Stockholm, Sweden; and ${ }^{2}$ NeuroSearch A/S, Ballerup, Denmark.

Background: Pridopidine (ACR16) belongs to a novel class of compounds called dopaminergic stabilizers. Initial studies in patients with Huntington's disease (HD) have been encouraging, demonstrating some beneficial effects, including improvement in motor function and affective symptoms. To further investigate the potential for pridopidine as a symptomatic treatment in HD, two clinical trials are ongoing.

Methods: The MermaiHD study (Multinational EuRopean Multicentre ACR16 study In HD) is a phase 3, double-blind, placebo-controlled study in eight European countries. Patients are randomized to pridopidine ( $45 \mathrm{mg}$ q.d. or $45 \mathrm{mg}$ b.i.d.) or placebo in equal proportions. This 6-month study is followed by a 6-month, open-label extension. The HART study (HD ACR16 Randomized Trial) is a double-blind, placebo-controlled, phase IIb study in the U.S. and Canada. It is of 3 months duration, and patients are randomly assigned to pridopidine $(10,22.5$, or $45 \mathrm{mg}$ b.i.d.) or placebo. The primary efficacy endpoint for both studies is the effect of pridopidine on patients' voluntary motor function as measured by the modified motor score-a subscale of the Unified HD Rating Scale. Secondary endpoints include the overall clinical impression of the patients, cognitive function, and the severity of neuropsychiatric symptoms.

Results: The MermaiHD study has recruited the targeted population of 420 patients. A substantial number of patients have finished the 6-month blinded treatment period and have now entered the open-label extension. Early results from the doubleblind MermaiHD study are expected to be available in early 2010 . 
Conclusions: Data from these studies will inform on the potential of pridopidine to improve symptoms in patients with HD.

\section{POSTER 21}

EURO-HDB-The First Large, Comprehensive European Study on the Burden of Huntington's Disease.

J. Dorey, ${ }^{1}$ J. Tedroff, ${ }^{2}$ M. Lamure, ${ }^{3}$ and M. Toumi. ${ }^{3}{ }^{1}$ Creativ Ceutical, Paris, France; ${ }^{2}$ NeuroSearch Sweden AB, Stockholm, Sweden; and ${ }^{3}$ University Claude Bernard Lyon 1, Villeurbanne, France.

Background: Understanding the determinants of health-related quality of life (HR-QoL) and cost is critical for medical doctors, health policy makers, and payers to guide management of disease. We initiated a large observational study to collect information on clinical characteristics, HR-QoL, and healthcare resource utilizations in France, Germany, Italy, Spain, Sweden, and the UK. The main aim of the study is to evaluate the cost of illness associated with Huntington's disease from various perspectives.

Methods: Patients with Huntington's disease are recruited through patient support organizations. The Huntington Self Assessment Instrument (HSAI), a fully self-reported patient questionnaire, has been developed and is complimented with SF-36 and EQ-5D questionnaires, the Zarit caregiver burden scale, and the Hospital Anxiety and Depression Scale (HADS) questionnaire. A country-specific medical cost dictionary will be developed, listing all unit costs for healthcare resource utilizations. Indirect costs associated with loss of productivity will be calculated using appropriate costing models for human capital methodology and all other aspects specific to Huntington's disease. The total cost of management from societal, health insurance, and patient perspectives will be calculated by multiplying resource utilizations by unit cost. Modeling of total costs will include presumed covariables and significant covariates from patients' socio-demographics using the Manning's algorithm, in order to identify the drivers of the various costs, depending on the perspective. Both kurtosis and heteroscedasticity will determine which model is to be used, and where the general linear model is chosen, the variance function will be selected using the Park test. Utility will be calculated using the self-reported EQ-5D questionnaire. The drivers of the utility will be identified using a classical ANOVA technique.

Conclusions: This study will provide valuable information for medical doctors, health policy makers, and payers to guide healthcare decisions.

\section{POSTER 22}

The Huntington Self-Assessment Instrument (HSAI): A Comprehensive and Specific Tool to Measure the Burden of Huntington's Disease.

J. Tedroff, ${ }^{1}$ J. Dorey, ${ }^{2}$ M. Lamure, ${ }^{3}$ J.-M. Graf von der Schulenburg, ${ }^{4}$ and M. Toumi. ${ }^{3}{ }^{1}$ NeuroSearch Sweden AB, Stockholm, Sweden; ${ }^{2}$ Creativ Ceutical, Paris, France; ${ }^{3}$ University Claude Bernard Lyon 1, Villeurbanne, France; and ${ }^{4}$ Institut für Versicherungsbetriebslehre, Leibniz Universität Hannover, Königsworther Platz 1, Hannover, Germany.

Background: Currently, data regarding the burden of Huntington's disease (HD) are scarce, and no specific self-reporting assessment instruments are available. There is a clear need for the design of self-reporting techniques that address patient reported outcomes (PRO), caregiver burden (CGB), and caregivers' healthcare resource utilization (RU) in HD.
Methods: We developed the Huntington Self-Assessment Instrument (HSAI), a tool that assesses PRO, CGB, and RU specifically in HD. The HSAI is split into two sections: the Huntington Self-Reported Instrument (H-SRI) and the Huntington Resources Utilization Interview (H-RUI). Each of the sections contain a questionnaire; one designed for the patient and the other for the caregiver. The patient section of the H-SRI is based on the Unified HD Rating Scale (UHDRS), with three parts that assess motor, behavioral, and functional capacity. It was critical to establish a self-reported assessment that would be a good proxy for the UHDRS clinical motor evaluation, an evaluation normally performed by an experienced practitioner. Visual analogue scales were therefore used to optimize patients' understanding of the questionnaire, and maximize assessment accuracy. The caregiver part of the H-SRI consists largely of the validated HD Quality of Life Battery for Carers (HDQoL-C). The patient part of the H-RUI assesses direct and indirect healthcare RU; the caregiver part determines the outof-pocket costs and the opportunity costs of patient care.

The HSAI will be available in English, German, French, Spanish, Italian, and Swedish; the Swedish version will be validated first. The UHDRS, Hospital Anxiety and Depression Scale (HAD), EQ-5D, SF-36, and the Zarit scale will be used for external validation of the HSAI. Internal validation will be carried out using standard methods. HSAI validation data will be presented at the meeting.

Conclusions: The HSAI is an important development that may be used to generate data required for appropriate policy on HD management.

\section{POSTER 23}

Pharmacology of the Dopaminergic Stabilizer Pridopidine (ACR16).

S. Waters, ${ }^{1}$ F. Pettersson, ${ }^{1}$ T. Dyhring, ${ }^{2}$ C. Sonesson, ${ }^{1}$ J. Tedroff, ${ }^{3}$ N. Waters, ${ }^{1}$ and H. Pontén. ${ }^{1}{ }^{I}$ NeuroSearch Sweden $A B$, Göteborg, Sweden; ${ }^{2}$ NeuroSearch A/S, Ballerup, Denmark; and ${ }^{3}$ NeuroSearch Sweden AB, Stockholm, Sweden.

Background: 4-[3-(methylsulfonyl)phenyl]-1-propylpiperidine hydrochloride (pridopidine, ACR16) is in development as an agent to target both the motor and behavioral symptoms of Huntington's disease (HD). Pridopidine is a dopaminergic stabilizer, a novel class of drugs that act at dopamine 2 (D2) receptors and display state-dependent behavioral effects. Preclinical studies in vivo suggest that pridopidine has potential efficacy across several clinical domains relevant for HD, including motor function, anxiety, depression, and psychosis. Early clinical studies with pridopidine have been encouraging in terms of reducing HD symptoms, without producing unwanted side effects.

Methods: Pridopidine was given subcutaneously to male Sprague-Dawley rats. HPLC was used to assess 3,4-dihydroxyphenylacetic acid (DOPAC) levels in brain tissue, and dopamine and noradrenaline levels in brain dialysates. Expression of activity-regulated cytoskeletal protein (Arc) was determined using RT-PCR on brain tissue RNA. Motility meters were used to quantify spontaneous and drug-induced locomotor activity. In vitro D2 radioligand binding and calcium-fluorescence functional studies were performed using human D2 expressing HEK293 cells.

Results: Pridopidine increased striatal tissue levels of the dopamine metabolite DOPAC $(\mathrm{ED} 50=81 \mu \mathrm{mol} / \mathrm{kg})$ and prefrontal cortex dialysate levels of dopamine and noradrenaline. Arc mRNA in the frontal cortex increased dose-dependently, indicating enhanced N-methyl-D-aspartate receptor-mediated signaling. Pridopidine reduced hyperlocomotion (d-amphet- 
amine: $\mathrm{ED} 50=40 \mu \mathrm{mol} / \mathrm{kg} ;$ MK801: ED50 = $54 \mu \mathrm{mol} / \mathrm{kg}$ ), but preserved spontaneous locomotor activity, confirming state-dependent behavioral effects. In vitro studies with pridopidine revealed surmountable low potency D2 antagonism, no intrinsic activity, and fast recovery of receptor-mediated responses.

Conclusions: Pridopidine stabilizes psychomotor activity by functional D2 antagonism and strengthening of cortical glutamate functions. Strengthening of cortical glutamatergic transmission may be key to the efficacious in vivo effects of pridopidine in states of hyperdopaminergia and hypoglutamatergia. The dopaminergic stabilizer pridopidine may offer clinical relief of psychomotor symptoms arising from dopaminergic dysfunction in HD.

\section{POSTER 24}

Worry and Experience of Genetic Discrimination in Persons at Risk for Huntington Disease.

C. Erwin, ${ }^{1}$ J. Williams, ${ }^{2}$ A. Juhl, ${ }^{3}$ J. Paulsen, ${ }^{3}$ and the Investigators of the Huntington Study Group. ${ }^{1}$ University of Texas Medical School at Houston, John P. McGovern Center for Health, Humanities and the Human Spirit, Department of Family Medicine, Houston, TX, USA; ${ }^{2}$ College of Nursing, University of Iowa, Iowa City, IA, USA; and ${ }^{3}$ College of Medicine, Department of Psychiatry, University of Iowa, Iowa City, IA, USA.

Background: Genetic discrimination (defined as the denial of rights, privileges, or opportunities, or other adverse treatment based solely on genetic information) is of concern to individuals at risk for developing Huntington disease (HD). Some prior surveys of genetic discrimination have included an examination of the prevalence of worry about genetic discrimination as contrasted to the prevalence of discriminatory experiences. The International RESPOND-HD (I-RESPOND-HD) survey compares and contrasts these two distinct psychological and experiential traits.

Methods: A total of 433 persons completed the I-RESPOND-HD survey examining the perceptions and experience of genetic discrimination. Participants had previously undergone predictive testing for the genetic mutation that causes HD (PREDICT-HD; $\mathrm{n}=310$ ), or had a $50 \%$ risk for developing HD due to family history but had chosen not to be tested (PHAROS; $\mathrm{n}=123$ ). Respondents lived in the United States ( $\mathrm{n}$ $=293)$, Australia $(\mathrm{n}=93)$, or Canada $(\mathrm{n}=46)$. Using qualitative and quantitative assessments, the survey instrument provides a comprehensive set of questions designed to elicit descriptions of the perception of genetic discrimination and how such discrimination might negatively influence respondents (worry).

Results: Participants self-report a much higher rate of worry about genetic discrimination than actual discriminatory events. Overall, $51.2 \%$ of respondents report worrying about the possibility of discrimination within relationships, but only $32.9 \%$ actually experienced such discrimination within their personal relationships. Participants similarly reported a higher rate of worry than actual discrimination in the context of insurance (70.0\% worried, $25.9 \%$ experienced), employment $(44.0 \%$ worried, $6.5 \%$ experienced), and in other transactions $(33.3 \%$ worried, $4.6 \%$ experienced).

Conclusion: Individuals at risk for HD worry about potential discrimination at a rate higher than that at which they experience such incidents. This exaggerated worry may motivate them to place subtle and/or overt limits on themselves, such as keeping their family history a secret, abstaining from genetic testing, or passing by opportunities to change employers.

\section{POSTER 25}

Longitudinal Analysis of the UHDRS in Individuals AtRisk for Huntington's Disease by Gene Status: The Prospective Huntington's Disease Observational At-Risk Study (PHAROS).

Huntington Study Group PHAROS Investigators . University or Rochester, Rochester, NY, USA.

Background: PHAROS (Arch Neurol 2006;63:991) aims to identify the earliest and most specific features associated with the motor diagnosis of Huntington disease (HD) among adults at risk for HD who are unaware of their gene status.

Methods: PHAROS investigators unaware of HD gene status assessed all domains of the Unified Huntington Disease Rating Scale (UHDRS) in PHAROS participants. Data were analyzed by CAG expanded $(\geq 37)$ versus nonexpanded $(<37)$. Subjects missing CAG data $(n=4)$ or with a motor diagnosis of $\mathrm{HD}$ (rated $\geq 99 \%$ diagnostic confidence) at baseline $(n=14)$ were excluded. Appropriate tests compared baseline demographics and UHDRS scores between the groups. A repeated measures analysis adjusted for age and gender was used to measure a divergence over time between groups. A secondary analysis excluded individuals that were ever given a motor diagnosis of HD.

Results: A total of 983 (345 expanded versus 638 nonexpanded) participants were analyzed. The mean duration of follow up was 5.5 ( \pm 2.6 ) years. At baseline, CAG $\geq 37$ participants had more impaired total motor $(p<0.0001)$, cognitive $(p$ $<0.05$ for all, except Verbal Fluency [ $p=0.52]$ ), and behavioral scores $(p<0.001)$, but not functional scores. Total motor, cognitive, and all functional scores significantly worsened over time in $\mathrm{CAG} \geq 37$ participants compared with $\mathrm{CAG}<37$ participants $(p<0.0001$ for all). Behavioral scores did not worsen over time between groups $(p=0.80)$. When participants with a motor diagnosis were excluded, the baseline differences and divergence in UHDRS scores were less pronounced but remained statistically significant for motor, cognitive, and functional UHDRS domains and were unchanged for behavior.

Conclusions: The UHDRS motor and cognitive domains show specificity for the HD CAG expansion at baseline and over time. All domains worsened over time in the $\mathrm{CAG} \geq 37$ group compared with the $<37$ group except behavior, while functional measures did not separate the groups at baseline.

\section{POSTER 26}

Word Reading Compared to Demographics-Based Estimates of Premorbid IQ in Early Huntington Disease.

W. Adams, J. Byars, J. O'Rourke, A. Flynn, J. Fiedorowicz, K. Duff, A. Leserman, P. Nopoulos, and L. Beglinger. University of Iowa, Iowa City, IA, USA.

Background: Irregular word pronunciation is a common method of estimating premorbid intellect, because it is resistant to neurological insult/deterioration. However, emerging research suggests word reading ability declines in some neurodegenerative diseases, resulting in an underestimation of premorbid IQ. Misrepresentation of predisease intellect may affect clinical diagnosis and confound the selection of participants for clinical trials. An alternative method is the use of a demographically-based equation to estimate intelligence.

Aim: To compare the WRAT Reading Subtest (a pronunciation measure) and the Barona Index (a demographically-based regression equation) as estimates of premorbid IQ in individuals with mild to moderate Huntington disease (HD).

Method: Using participants $(n=73)$ pooled from two clinical trials and an outpatient HD clinic, we examined WRAT and 
Barona standard scores and their discrepancy, along with demographic and clinical characteristics.

Results: Mean age was 47.6 years $(\mathrm{SD}=11.7)$, total functional capacity (TFC) was 9.6 ( $\mathrm{SD}=3.0$ ), and total motor score (TMS) was $32.7(\mathrm{SD}=15.8)$. Average WRAT standard score was $91.9(\mathrm{SD}=11.9)$, while Barona was $108.0(\mathrm{SD}=7.2)$, with a mean discrepancy of $16.1(\mathrm{SD}=10.7$; range -42.3 to +6.3 ). Although true premorbid IQ is unknown, this sample overrepresented higher levels of education (i.e., $\sim 22 \%$ of the sample had $16+$ years, while $\sim 8 \%$ had $<12$ years). Pearson correlations revealed that only WRAT reading was associated with motor functioning $(\mathrm{r}=-0.245, p<0.05)$. Neither measure correlated with functional capacity.

Discussion: The WRAT produced premorbid IQ estimates lower than the Barona for $93 \%$ of participants and was below expectations given the sample's mean education. Unlike the Barona, the WRAT was associated with impaired motor functioning, suggesting that it may decline with disease. Therefore, the Barona Index may be a more appropriate measure of premorbid intelligence in persons with HD and should be considered for subject selection in clinical trials and clinical evaluation.

\section{POSTER 27}

Characterizing Psychomotor Declines in Prodromal Huntington Disease with the Trail Making Test.

J. O'Rourke, ${ }^{1}$ L. Beglinger, ${ }^{1}$ J. Mills, ${ }^{1}$ M. Smith, ${ }^{1}$ J. Stout,${ }^{2}$ S. Queller, ${ }^{3}$ S. Rao, ${ }^{4}$ J. Zimbelman, ${ }^{4}$ and J. Paulsen. ${ }^{1}{ }^{1}$ University of Iowa, Iowa City, IA, USA; ${ }^{2}$ Monash University, Melbourne, Australia; ${ }^{3}$ Indiana University, Bloomington, IN, USA; and ${ }^{4}$ Cleveland Clinic, Cleveland, $\mathrm{OH}, \mathrm{USA}$.

Objective: Individuals who have the Huntington disease (HD) gene-expansion but do not yet meet clinical criteria for HD (prHD) demonstrate detectable psychomotor declines long before diagnosis. The aim of this study is to assess performance on the Trail Making Test (TMT), a well known neuropsychological measure of psychomotor speed and aspects of attention and executive functioning in prHD. A secondary aim is to determine which aspects of preclinical motor decline affect TMT performance.

Method: A total of 984 prHD participants and non-gene expanded controls from the PREDICT-HD study were assessed with the TMT and the motor rating scale within the Unified Huntington's Disease Rating Scale (UHDRS). The TMT consists of 2 parts: TMT-A, a measure of psychomotor speed and attention, and TMT-B, which adds a cognitive set-shifting component. Index scores were seconds to complete TMT-A and TMT-B, as well as the difference between the two parts. The UHDRS motor scale was analyzed using the 5 motor factors (i.e., oculomotor, dystonia, rigidity, bradykinesia, and chorea) identified by Marder et al. (2000). PrHD participants were grouped based on estimated proximity to diagnosis (NEAR, $<9$ years; MID 9-15 years, FAR $>15$ years) according to CAG repeat length and age, using Langbehn et al.'s (2004) formula. Results: Linear modeling and post-hoc comparisons revealed differences between all prognostic groups, with the NEAR group performing worst on all three TMT measures $(p<0.01)$. The FAR group was not significantly different from controls. Stepwise regression results showed associations for bradykinesia and dystonia with aspects of TMT performance.

Conclusion: PrHD participants $\leq 15$ years to diagnosis are different from controls on all TMT measures, which indicates that TMT might be a sensitive outcome measure in preventative clinical trials. Bradykinesia was most consistently associated with poorer performance, suggesting that slowed performance, rather than oculomotor changes or chorea, are most related to TMT performance.

\section{POSTER 28}

Delphi Process for the Development of Treatment Guidelines for Behavioral Symptoms and Chorea in Huntington's Disease.

E. van Duijn, ${ }^{1}$ M. Groves, ${ }^{2}$ D. Craufurd,${ }^{3}$ K. Anderson, ${ }^{4}$ M. Guttman, ${ }^{5}$ E. Wexler, ${ }^{6}$ S. Perlman, ${ }^{7}$ J.-M. Burgunder, ${ }^{8}$ A. Rosenblatt, ${ }^{9}$ D. van Kammen, ${ }^{10}$ J. Giuliano, ${ }^{10}$ and L. Goodman. ${ }^{11}{ }^{1}$ Leiden University Medical Center, Psychiatry, Leiden, The Netherlands; ${ }^{2}$ Beth Israel Medical Center, Psychiatry, New York, NY, USA; ${ }^{3}$ Manchester University, Psychiatry, Manchester, UK; ${ }^{4}$ University of Maryland, Dept. of Neurology and Dept. of Psychiatry, Baltimore, MD, USA; ${ }^{5}$ The Centre for Movement Disorders, Markham, Ontario, Canada; ${ }^{6}$ Dept. of Psychiatry \& Biobehavioral Sciences, UCLA Center for Neurobehavioral Genetics, Los Angeles, CA, USA; ${ }^{7}$ University of California, Los Angeles, Los Angeles CA, USA; ${ }^{8}$ Neurologische Klinik und Poliklinik, Universitätsspital Bern, Bern, Switzerland; ${ }^{9}$ Dept. of Psychiatry, Johns Hopkins University, Baltimore, MD, USA; ${ }^{10}$ Cure Huntington's Disease Initiative (CHDI), Princeton, NJ, USA; and ${ }^{11} H D$ Drug Works, Lake Forest Park, WA, USA.

Background: Despite large gaps in evidence-based knowledge, clinical experience supports the use of pharmacologic treatments for many symptoms of Huntington's disease (HD). The project goal is to develop consensus guidelines based on expert clinical experience that will improve the quality of HD care.

Methods/Techniques: The Delphi process, which provides an iterative formal strategy to gather expert opinion and form a consensus, was utilized. First steps of this process explored expert prescribing patterns. Step 1: Nine experts from Huntington's Study Group and European HD Network created initial statements for irritability, obsessive/compulsive behaviors $(\mathrm{O} /$ $\mathrm{Cs}$ ), and chorea. Step 2: Statements were submitted via a web survey to a panel of 40 additional experts.

Results: Thirty expert neurologists and psychiatrists from Europe and North America responded. For irritability there was lack of consensus in first choice and in sequence of drugs used in combination therapy. For $\mathrm{O} / \mathrm{Cs}$ there was high agreement $(>80 \%)$ on SSRI as first choice; however, there was lack of consensus on adjuvant drug therapy. For chorea there was agreement that it should be treated when quality of life or ADL is affected, but there was lack of consensus on drug of first choice. The next round of the Delphi consensus process will begin in November of 2009.

Conclusions: Many areas of variability in the treatment for irritability, O/Cs, and chorea in HD, even among expert clinicians, have been identified. Results will guide future rounds of the Delphi process to elicit rational causes for the differences identified. Collection of further data on consensus will begin in November of 2009. When complete, this process will clarify useful treatment paradigms that will improve patient care and identify areas in which clinical trials would be most useful.

\section{POSTER 29}

Multivariate Clinical Predictors of Huntington Disease (HD): Prospective Results from the PREDICT-HD Study. D. Langbehn, J. Paulsen, and the PREDICT-HD Investigators of the Huntington Study Group. University of Iowa, Iowa City, IA, USA.

For text of abstract, see platform presentation (above, 12:25 $-12: 40$ PM). 